\title{
LAPPED TIGHT FRAME TRANSFORMS
}

\author{
Amina Chebira ${ }^{1}$ and Jelena Kovačević ${ }^{1,2}$ \\ ${ }^{1}$ Dept. of Biomedical Eng. and Center for Bioimage Informatics, ${ }^{2}$ Dept. of Electrical and Computer Eng. \\ Carnegie Mellon University, Pittsburgh, PA \\ Email: amina@cmu.edu, jelenak@cmu.edu
}

\begin{abstract}
We propose a new class of equal-norm tight frames termed Lapped Tight Frame Transforms (LTFTS). These can be seen as a redundant counterpart to bases known as Lapped Orthogonal Transforms (LOTs) introduced by Malvar and Cassereau, as well as an infinitedimensional counterpart to Harmonic Tight Frames (HTFs). To construct LTFTs, we seed them from LOTs and show that, in a specific case, the process preserves the equal norm. As both their basis counterpart LOTs as well as their finite-dimensional one HTFs, LTFTs possess many desirable properties, such as equal norm and efficient implementation.
\end{abstract}

Index Terms - Wavelets, frames, filter banks, lapped orthogonal transforms, harmonic tight frames.

\section{INTRODUCTION AND MOTIVATION}

Redundancy, a common tool in signal processing and communications, has found its way into signal representations. Over the past few years, many applications have taken advantage of such redundant, signal representations, such as robust transmission, denoising, quantization and many others (see [1] and references therein). The nonredundant representations are bases; their redundant counterparts are called frames. Frames were originally introduced by Duffin and Schaeffer [2], and popularized later on in $[3,4,5,6]$.

A particular class of interest are tight frames (TFs) which can be seen as a generalization of orthonormal bases (ONBs). Tight frames are sought after for the same reasons orthonormal bases are; they are self-dual, efficient to compute and they preserve the norm. Typically, tightness is imposed when one needs to reconstruct and stability of reconstruction is an issue. Since TFs do not require inversion of matrices, they seem a natural choice. In finite dimensions, TFs are linearly dependent sets of vectors allowing more freedom in design than ONBs (release of orthogonality constraint). However, this freedom comes at a price, and depending on the application at hand, the amount of redundancy is a design criterion. On top of the tightness constraint, sought-after qualities are for all frame vectors to be of equal norm (equal-norm tight frames-ENTFs) and maximum robustness (MR), the property which allows the loss of all frame coefficients but the necessary number for reconstruction.

In an ever-continuing search for new frame families, an appealing option is the process of obtaining TFs from ONBs in larger dimensions, known as the Naimark Theorem [7], or, seeding [8]. All tight frames can be obtained this way. In this paper, we seed LOTs to obtain a new class of frames termed Lapped Tight Frame Transforms

This work was supported by NSF through awards CCF-0515152 and EF-0331657 as well as by the PA State Tobacco Settlement, Kamlet-Smith Bioinformatics Grant.
(LTFTS). LOTs were introduced by Cassereau [9] and Malvar [10] in response to one of the major drawbacks of the DCT-the blocking effect created by processing of the signal block by block. The LOTs solve the problem by processing blocks of overlapping data. LOTs can be viewed as a particular case of a perfect reconstruction filter bank with $m$ channels and filter length $l=2 m$ and basis functions being either cosines $[10,11]$ or complex exponentials [12]. The length $l$ was generalized to any multiple integer of $m$ in [10].

Obtained by seeding, the LTFTs can be seen both as the frame counterpart of LOT bases as well as the infinite-dimensional, filterbank counterpart of the most famous frame family-Harmonic Tight Frames (HTFs, seeded from the DFT). These relationships are illustrated below.

\begin{tabular}{lccc}
\hline & $\begin{array}{c}\text { finite-dimensional } \\
\text { (block transforms) }\end{array}$ & $\begin{array}{c}\text { infinite-dimensional } \\
\text { (overlapped transforms) }\end{array}$ \\
\hline ONBs & DFT & $\rightarrow$ & LOT \\
& $\downarrow$ & & $\downarrow$ \\
TFs & HTF & $\rightarrow$ & LTFT \\
\hline
\end{tabular}

There has already been some work done in designing LTFTs. In particular, in [13], the authors propose a LTFT derived from the extended lapped complex transform [12]. These are not obtained by seeding (they start from a frame) and while are in spirit similar to what we are proposing, they lead to a completely different family. The same authors have already proposed a 2D nonseparable LTFT.

Organization of the paper. In Section 2, we give an overview of frames, starting with frames in finite dimensions. We then introduce a class of infinite-dimensional frames, those implementable via filter banks. In Section 3, we give a brief review of LOTs and then follow by presenting the construction of LTFTs. For a specific LOT family, seeding a specific LTFT we call Princen-Bradley LTFT, we prove that the LTFTs are equal-norm (tightness is guaranteed by construction) and discuss issues related to window design.

\section{FILTER BANK FRAMES}

We start with a brief account of signal representations in finite dimensions and then follow up with how signals are represented using frames both in finite and infinite dimensions.

Assume finite-dimensional spaces $\mathbb{R}^{m}$ or $\mathbb{C}^{m}$. Given an ONB for such a space, $\Psi=\left\{\psi_{i}\right\}_{i=0}^{m-1}$, we associate to it a matrix (opera- 
tor) which we will also call $\Psi$ :

$$
\Psi=\left(\begin{array}{ccc}
\psi_{0,0} & \cdots & \psi_{m-1,0} \\
\vdots & \ddots & \vdots \\
\psi_{0, m-1} & \cdots & \psi_{m-1, m-1}
\end{array}\right)
$$

Matrix $\Psi$ has basis vectors as its columns, and $\psi_{i, j}$ is the $j$ th element of the $i$ th basis vector. Then, a signal $x$ belonging to $\mathbb{R}^{m}$ or $\mathbb{C}^{m}$ can be expressed as:

$$
x=\Psi X=\Psi \Psi^{*} x,
$$

where $X$ is the vector from $\mathbb{R}^{m}$ or $\mathbb{C}^{m}$ of so-called transform coefficients (inner products), and $\Psi^{*}$ denotes the Hermitian transpose of $\Psi$. Of course, the above implies that $\Psi \Psi^{*}=I$, which further implies that $\Psi$ is a unitary matrix and thus represents an ONB.

\subsection{Finite-Dimensional Frames}

The above is true for frames as well, except that the number of frame vectors $m$ is larger or equal than the dimension of the space $n$. We will cover finite-dimensional frames through an example; the so-called Mercedes-Benz frame. It is the simplest and best known frame, has 3 vectors in 2 dimensions and is an example of a harmonic tight frame. Its frame operator is now a rectangular matrix and is given by

$$
\Phi=\left(\begin{array}{lll}
\varphi_{1} & \varphi_{2} & \varphi_{3}
\end{array}\right)=\left(\begin{array}{ccc}
0 & -1 / \sqrt{2} & 1 / \sqrt{2} \\
\sqrt{2 / 3} & -1 / \sqrt{6} & -1 / \sqrt{6}
\end{array}\right) .
$$

Similarly to bases, one can check that the above frame expands signals in $\mathbb{R}^{2}$ as

$$
x=\Phi X=\Phi \Phi^{*} x .
$$

As before, the above implies that $\Phi \Phi^{*}=I$, and the corresponding frame is tight. Moreover, it is an equal-norm tight frame (ENTF) since all three frame vectors have the same norm $\sqrt{2 / 3}$.

Tight frames are prized for the same good properties ONBs possess, one of them being norm preservation. (We can easily check that the MB frame preserves the norm.) Actually, tight frames are redundant sets of vectors closest to ONBs.

HTFs are obtained by seeding the DFT, where by seeding we will mean the following: A frame $\Phi$ is obtained by seeding from a basis $\Psi$ by deleting a suitable set of columns of $\Psi$ [8], denoted by

$$
\Phi^{*}=\Psi[J],
$$

where $J \subset\{1, \ldots, m\}$ is the index set of the retained columns.

Since the MB frame is the simplest example of an HTF, it, too, must have been obtained by seeding. To see seeding in action, start with the matrix $\Psi$ unitarily equivalent to the real DFT in 3 dimensions:

$$
\Psi=\left(\begin{array}{ccc}
0 & \sqrt{2 / 3} & 1 / \sqrt{3} \\
-1 / \sqrt{2} & -1 / \sqrt{6} & 1 / \sqrt{3} \\
1 / \sqrt{2} & -1 / \sqrt{6} & 1 / \sqrt{3}
\end{array}\right)
$$

and use the following projection operator $P$ :

$$
P=\frac{1}{\sqrt{3}}\left(\begin{array}{ccc}
2 / 3 & -1 / 3 & -1 / 3 \\
-1 / 3 & 2 / 3 & -1 / 3 \\
-1 / 3 & -1 / 3 & 2 / 3
\end{array}\right)
$$

to obtain the MB frame as in (1). Thus, (1) is the MB frame seen as a collection of vectors in the three-dimensional space and $\Phi_{3 \mathrm{D}}^{*}=$ $P \Psi$. The projection operator essentially "deletes" the last column of $\Psi$ to create the frame $\Phi^{*}$.

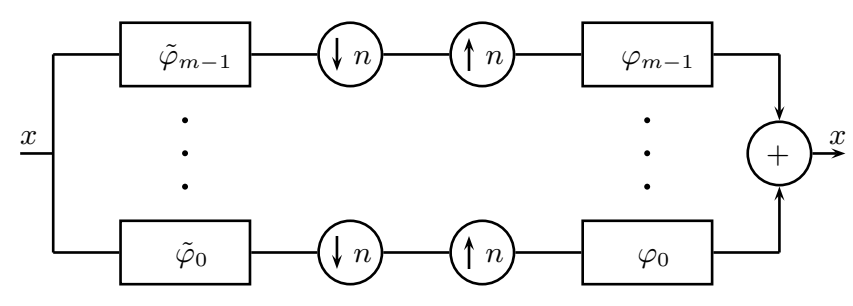

Fig. 1. A filter-bank implementation of a frame expansion: It is an $m$-channel filter bank with sampling by $n$.

\subsection{Infinite-Dimensional Frames Via Filter Banks}

The only infinite-dimensional class of frames we discuss are those implemented by filter banks (FBs), the reason being that these are frames used in applications and our only link to the real world. The vectors (signals) live in the infinite-dimensional Hilbert space $\ell^{2}(\mathbb{Z})$. In fact, we can investigate finite-dimensional frames within the filter bank framework as well. In other words, all cases we consider, both finite-dimensional and infinite-dimensional, we can look at as filter banks. A thorough analysis of filter banks frames is given in $[14,15]$.

A filter bank (FB) is the basic signal processing structure used to implement most multiresolution transforms. Fig. 1 depicts a filter bank with $m$ channels and sampling by $n$. If $m=n$, we deal with critically-sampled filter banks implementing bases, while if $m>n$, we deal with oversampled filter banks implementing frames. In the figure, a general frame is given. We will see in a moment, that for a tight frame, $\tilde{\varphi}_{i}=\varphi_{i}$.

As in (2), we can express the filter-bank frame decomposition as

$$
x=\Phi X=\Phi \Phi^{*} x
$$

where $x$ is now an infinite sequence belonging to $\ell^{2}(\mathbb{Z}), X$ is an infinite sequence of transform coefficients (inner products), and $\Phi$ is the TF expansion matrix. Assume that the nonzero support of the filter $\varphi_{i}$, or, its length is $l=k n$ (if not, we can always pad with zeros), and write the frame operator as

$$
\Phi=\left(\begin{array}{ccccccc}
\ddots & \vdots & \vdots & \vdots & \vdots & \vdots & \ddots \\
\cdots & \Phi_{0} & 0 & \cdots & 0 & 0 & \cdots \\
\cdots & \Phi_{1} & \Phi_{0} & \cdots & 0 & 0 & \cdots \\
\cdots & \vdots & \vdots & \vdots & \vdots & \vdots & \ldots \\
\cdots & \Phi_{k-1} & \Phi_{k-2} & \cdots & \Phi_{0} & 0 & \cdots \\
\cdots & 0 & \Phi_{k-1} & \cdots & \Phi_{1} & \Phi_{0} & \cdots \\
\ddots & \vdots & \vdots & \vdots & \vdots & \vdots & \ddots
\end{array}\right),
$$

where each block $\Phi_{r}$ is of size $n \times m$ :

$$
\Phi_{r}=\left(\begin{array}{ccc}
\varphi_{0, r n} & \cdots & \varphi_{m-1, r n} \\
\vdots & \ddots & \vdots \\
\varphi_{0, r n+n-1} & \cdots & \varphi_{m-1, r n+n-1}
\end{array}\right) .
$$

We can rephrase the frame decomposition in the $z$-domain as well, where a filter bank implements a TF decomposition in $\ell^{2}(\mathbb{Z})$ if and only if its polyphase matrix $\Phi_{p}(z)$ is paraunitary [14]. A polyphase matrix $\Phi_{p}(z)$ collects the $z$-domain versions of subsequences modulo $n$. For frames, $\Phi_{p}(z)$ is of size $n \times m$ and can be written as:

$$
\Phi_{p}(z)=\sum_{r=0}^{k-1} \Phi_{r} z^{-r}
$$


where $\Phi_{r}$ are as defined in (7). A paraunitary polyphase matrix satisfies

$$
\Phi_{p}(z) \Phi_{p}^{*}(z)=c I
$$

where $\mathrm{c}$ is a constant. When the filter length $l$ is equal to the sampling factor $n$, we have a block transform. Then, in (6), only $\Phi_{0}$ is nonzero, making $\Phi$ block-diagonal. In effect, since there is no overlap between processed blocks, this can be analyzed as a finitedimensional case, where both the input and the output are $n$-dimensional vectors. This discussion shows how finite-dimensional frames can be analyzed in the filter-bank context.

\section{LAPPED TIGHT FRAME TRANSFORMS}

\subsection{Review of Lapped Orthogonal Transforms}

The LOTs can be seen as a class of $m$-channel filter banks implementing bases, originally developed for filters of length $l=2 \mathrm{~m}$ and later generalized to arbitrary integer multiples of $m$ [10]. The development of the LOT was spurred by the problem of blocking effect introduced in transform coding by block transforms such as the DFT and DCT. Compared to block transforms, the LOT keeps the same number of filters but doubles their length, which means that the basis functions of adjacent blocks overlap by half their size, thus removing the blocking effects. However, LOTs are not solely determined by their length, but by the specific form of their basis vectors as well. Two main classes of LOTs exist distinguished by whether they use cosines or complex exponentials in their basis functions; (a) Cosines were used in the LOTs introduced in $[9,10]$ (based on the DCT); we call those LOTs Cassereau-Malvar LOTs. Cosines were also used in [11] (modulated LOTs) and we call those LOTs Princen-Bradley LOTs. (b) Complex exponentials were used in [12] and we call these LOTs Young-Kingsbury LOTs.

In general, for a FB with length $l=2 m$, the time-domain matrix $\Psi$ has a double diagonal, that is, in (6), only $\Phi_{0}$ and $\Phi_{1}$ exist:

$$
\Psi=\left(\begin{array}{cccc}
\ddots & \vdots & \vdots & \ddots \\
\cdots & \Psi_{0} & 0 & \cdots \\
\cdots & \Psi_{1} & \Psi_{0} & \cdots \\
\cdots & 0 & \Psi_{1} & \cdots \\
\ddots & \vdots & \vdots & \ddots
\end{array}\right)
$$

Thus, (8) reduces to

$$
\Psi_{p}(z)=\Psi_{0}+z^{-1} \Psi_{1},
$$

where $\Psi_{r}, r=0,1$, are $m \times m$ matrices with $\left(\Psi_{r}\right)_{j, i}=\psi_{i, j}$ for $i=0, \ldots, m-1$ and $j=m r, \ldots m r+m-1$.

Since the LOT is a unitary transform, that is, $\Psi \Psi^{*}=\Psi^{*} \Psi=I$ the following must be satisfied:

$$
\begin{aligned}
& \Psi_{0} \Psi_{0}^{*}+\Psi_{1} \Psi_{1}^{*}=\Psi_{0}^{*} \Psi_{0}+\Psi_{1}^{*} \Psi_{1}=I, \\
& \Psi_{0}^{*} \Psi_{1}=\Psi_{1}^{*} \Psi_{0}=0, \quad \Psi_{0} \Psi_{1}^{*}=\Psi_{1} \Psi_{0}^{*}=0,
\end{aligned}
$$

where $I$ is an $m \times m$ identity matrix. This is valid for any of the LOT families defined above.

An example LOT is the Princen-Bradley LOT [11]:

$$
\psi_{i, j}=\sqrt{\frac{1}{m}} \cos \left(\frac{\pi(2 i+1)(2 j-m+1)}{4 m}\right),
$$

for $i=0, \ldots, m-1$ and $j=0, \ldots, 2 m-1$. Thanks to the particular structure of the cosines, the following is true

$$
\Psi_{0} \Psi_{0}^{*}=\frac{1}{2}(I-J), \quad \Psi_{1} \Psi_{1}^{*}=\frac{1}{2}(I+J),
$$

where $J$ is the anti-diagonal matrix.

With this construction, similarly to the DFT, we will have fixed basis functions allowing no freedom in design. To obtain a better design, one can add a window that multiplies each filter resulting in a modulated FB over the frequency band. This modulated FB can be modeled as $W \Psi$, where the window $W=\operatorname{diag}\left\{w_{j}\right\}_{j=0}^{2 m-1}$, and it is symmetric $w_{j}=w_{2 m-1-j}, j=0, \ldots 2 m-1$. Now, the perfect reconstruction conditions in (12) become

$$
W \Psi_{0} \Psi_{0}^{*} W+J W J \Psi_{1} \Psi_{1}^{*} J W J=I .
$$

Substituting (15) into (16), we obtain

$$
\frac{1}{2}\left(W^{2}+J W^{2} J\right)=I,
$$

which implies that the window has to satisfy a power-complementary property, that is $w_{j}^{2}+w_{m-1-j}^{2}=2$, for $j=0, \ldots, m-1$.

\subsection{Lapped Tight Frame Transform}

We previously mentioned that the HTFs are the counterpart of the DFT, that is, they are obtained by seeding the DFT. HTFs have a number of interesting properties: (a) They are efficient to compute (inherited from the DFT). (b) They are the only ENTF family whose elements are generated by a group of unitary operators with one prototype element. (c) For $m=n+1$, all ENTFs are unitarily equivalent to the HTF of the same size, and thus, all ENTFs for $m=n+1$ are known. (d) HTFs are maximally robust to erasures.

As we said, the HTFs are finite-dimensional frames and thus equivalent to block transforms. For the same reasons LOTs were introduced, we would like to find filter-bank frames seeded from the LOTs in the hope they will inherit all the good properties LOTs possess. It is important to note that in filter-bank parlance, seeding is done on the polyphase matrix. Suppose that $\Psi_{p}(z)$ is the $m \times m$ polyphase matrix associated with the DFT of size $m$. Then $\Psi_{p}(z)=\Psi_{0}$ and

$$
\Phi_{p}^{*}(z)=\Phi_{0}^{*}=\Psi_{p}[J]
$$

is the transpose of the HTF matrix. It turns out that the indices in $J$ do not have to be contiguous for the following discussion to hold, that is, we can erase any subset of $m-n$ columns from $\Psi_{p}(z)$ and still get an HTF. However, to simplify the discussion, we take $J=[0, \ldots, n-1]$. Note that for $m=3$ and $n=2$, this procedure leads to the MB frame described in Section 2.1 (within unitary equivalence).

Now, let us start with $\Psi_{p}(z)$ being the $m \times m$ polyphase matrix associated with the LOT of size $m$. Then (11) holds and $\Phi_{p}^{*}(z)=$ $\Phi_{0}^{*}+z^{-1} \Phi_{1}^{*}=\Psi_{p}[0, \ldots, n-1]$. The matrices $\Phi_{r}^{*}$ are now rectangular of size $n \times m$. For $r=0,1$, we have

$$
\Phi_{r}=\left(\begin{array}{ccc}
\psi_{0, m r}^{*} & \cdots & \psi_{0, m r+m-1}^{*} \\
\psi_{1, m r}^{*} & \cdots & \psi_{1, m r+m-1}^{*} \\
\vdots & \cdots & \vdots \\
\psi_{n-1, m r}^{*} & \cdots & \psi_{n-1, m r+m-1}^{*}
\end{array}\right)
$$

By Naimark Theorem, we know that this family is a TF, which implies that $\Phi_{p}(z) \Phi_{p}^{*}(z)=c I$ ( $c$ is a constant). Note that as opposed to the LOT case, the matrix products do not commute anymore.

All of the above is general and can be applied to any type of LOT. Let us now see through an example what happens when the obtained LTFT has been seeded by the Princen-Bradley LOT in (14). Seeding both the Cassereau-Malvar as well as the Young-Kingsbury 
LOTs is left for future work. For Princen-Bradley LOTs we can compute $\operatorname{diag}\left(\Phi_{0}^{*} \Phi_{0}+\Phi_{1}^{*} \Phi_{1}\right)_{i}=\left\|\varphi_{i}\right\|^{2}$. Note that

$$
\left\|\varphi_{i}\right\|^{2}=\sum_{j=0}^{n-1} \psi_{j, i}^{* 2}+\psi_{j, i+m}^{* 2}
$$

After some algebraic manipulations on the cosines, we can prove that

$$
\left\|\varphi_{i}\right\|^{2}=\frac{n}{m}, \quad i=0, \cdots, m-1,
$$

that is, the LTFT obtained is equal-norm.

If we start with the Princen-Bradley LOT with a window, and seed $W \Psi$, the tight frame obtained would loose its equal-norm property since $\left\|\varphi_{i}\right\|^{2}=(n / m) w_{i}^{2}$. To preserve equal norm, we have to modulate directly the LTFT after seeding the LOT. In the PrincenBradley LOTs, the window chosen was symmetric, that is, $w_{i}=$ $w_{2 m-1-i}$. We lift this restriction initially and assume a general window represented by a matrix $D$, a $2 n \times 2 n$ diagonal matrix. We can write $D=\left[\begin{array}{ll}D_{0} & D_{1}\end{array}\right]$ and $D_{r}$ is a $n \times n$ diagonal matrix. Unlike for the LOTs, the matrix product $\Phi_{0} \Phi_{0}^{*}$ has no particular structure, in fact,

$$
\left(\Phi_{0} \Phi_{0}^{*}\right)_{i, j}=a_{i, j}=\frac{1}{2 m} \frac{\sin \left(\frac{\pi(i+j+1)}{2}\right)}{\sin \left(\frac{\pi(i+j+1)}{2 m}\right)}+\frac{1}{2 m} \frac{\sin \left(\frac{\pi(i-j)}{2}\right)}{\sin \left(\frac{\pi(i-j)}{2 m}\right)} .
$$

Substituting this into (12), we obtain the following:

$$
\begin{gathered}
a_{j, j} d_{j}^{2}+\left(1-a_{j, j}\right) d_{n+j}^{2}=1, \\
d_{j} d_{s}=d_{n+j} d_{n+s}, \quad s=0, \cdots, n-1, s \neq j .
\end{gathered}
$$

The set of solutions to (19)-(20) is infinite. Of course, the constant window with $d_{j}=1$, for $j=0, \ldots, 2 n-1$ is also a solution to the above. Finding the best window amongst all the possible solutions is part of our future work.

If the window is symmetric, then (12) becomes:

$$
\begin{aligned}
D_{0} \Phi_{0} \Phi_{0}^{*} D_{0}+J D_{0} J \Phi_{1} \Phi_{1}^{*} J D_{0} J & =I \\
\text { with } \Phi_{0} \Phi_{0}^{*}+\Phi_{1} \Phi_{1}^{*} & =I .
\end{aligned}
$$

Using (21), we derive the following conditions on $D$ :

$$
\begin{gathered}
a_{j, j} d_{j}^{2}+\left(1-a_{j, j}\right) d_{n-j-1}^{2}=1, \\
d_{j} d_{s}=d_{n-j-1} d_{n-s-1}, \quad s=0, \cdots, n-1, s \neq j .
\end{gathered}
$$

Fixing $d_{0}=-1$, we have $d_{n-1}= \pm 1$ and $d_{s}=-d_{n-1} d_{n-s-1}$ for $s=1, \ldots, n-2$. Note that the same conditions hold for an antisymmetric window, that is, the half-windows can only be symmetric or antisymmetric. For a symmetric window, a possible solution, depicted in Fig. 2, is given by

$$
d_{j}=\left\{\begin{array}{ll}
\cos \left(\frac{j \pi}{n-1}+\pi\right) & \text { if } n \text { is even, } \\
\cos \left(\frac{2 j \pi}{n-1}+\pi\right) & \text { if } n \text { is odd, }
\end{array} \quad j=0, \ldots, n-1 .\right.
$$

Optimization of window design is left for future work. Since LTFTs are the counterparts of HTFs, we expect them to be maximally robust (MR) as well. This requirement arose in using frames for robust transmission where the loss of up to $m-n$ transform coefficient over the transmission channel would not be fatal. The loss of coefficients translates into removal of the corresponding set of $m-n$ columns in $\Phi_{p}(z)$ and the ability to reconstruct despite the loss translates into the remaining matrix being invertible. Hence, a LTFT is MR if and only if any $n \times n$ submatrix of $\Phi_{p}(z)$ is of full rank on the unit circle. We tested this on the LTFT example presented here for $m=4$ and $n=2$, where the MR property is more likely to fail, and the results were encouraging as it turned out that this family of LTFTs is MR for $m=4$ and $n=2$. We are currently working on a more general proof.
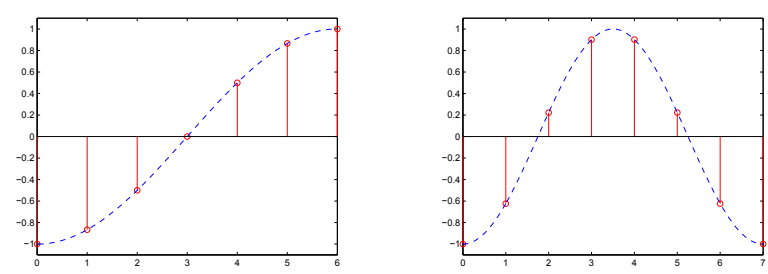

Fig. 2. Window solution to (23)-(24) for $n=7,8$ (left to right).

\section{REFERENCES}

[1] J. Kovačević and A. Chebira, "Life beyond bases: The advent of frames," IEEE Signal Proc. Mag., 2007.

[2] R. J. Duffin and A. C. Schaeffer, "A class of nonharmonic Fourier series," Trans. Amer. Math. Soc., vol. 72, pp. 341-366, 1952.

[3] I. Daubechies, A. Grossman, and Y. Meyer, "Painless nonorthogonal expansions," Journ. Math. Phys., vol. 27, pp. 1271-1283, Nov. 1986.

[4] O. Christensen, Introduction to Frames and Riesz Bases, Birkhäuser, 2002.

[5] D. Han and D. R. Larson, Frames, bases and group representations, Number 697 in Memoirs AMS. American Mathematical Society, Providence, RI, 2000.

[6] P. G. Casazza, "The art of frame theory," Taiwanese Journ. of Math., vol. 4, no. 2, pp. 129-202, 2000.

[7] N. I. Akhiezer and I. M. Glazman, Theory of Linear Operators in Hilbert Spaces, vol. 1, Frederick Ungar, 1966.

[8] M. Püschel and J. Kovačević, "Real, tight frames with maximal robustness to erasures," in Proc. Data Compr. Conf., Snowbird, Utah, Mar. 2005, pp. 63-72.

[9] P. M. Cassereau, "A new class of optimal unitary transforms for image processing," M.S. thesis, Massachusetts Institute of Technology, May 1985.

[10] H. S. Malvar, Signal Processing with Lapped Transforms, Artech House, Norwood, MA, 1992.

[11] J. Princen, A. Johnson, and A. Bradley, "Subband transform coding using filter bank designs based on time domain aliasing cancellation," in Proc. IEEE Int. Conf. Acoust., Speech and Signal Proc., Dallas, TX, Apr. 1987, pp. 2161-2164.

[12] R. W. Young and N. G. Kingsbury, "Frequency-domain motion estimation using a complex lapped transform," IEEE Trans. Image Proc., vol. 2, no. 1, pp. 2-17, Jan. 1993.

[13] J. Gauthier, L. Duval, and J.-C. Pesquet, "Low redundancy oversampled lapped transforms and application to 3D seismic data filtering," in Proc. IEEE Int. Conf. Acoust., Speech and Signal Proc., Toulouse, France, May 2006, vol. II, pp. 821824.

[14] Z. Cvetković and M. Vetterli, "Oversampled filter banks," IEEE Trans. Signal Proc., vol. 46, no. 5, pp. 1245-1255, May 1998.

[15] H. Bölcskei, F. Hlawatsch, and H. G. Feichtinger, "Frametheoretic analysis of oversampled filter banks," IEEE Trans. Signal Proc., vol. 46, no. 12, pp. 3256-3269, Dec. 1998. 